\title{
Article \\ Critical Comparison of Spherical Microindentation, Small Punch Test, and Uniaxial Tensile Testing for Selective Laser Melted Inconel 718
}

\author{
Zachary S. Courtright ${ }^{1,2}$, Nicolas P. Leclerc ${ }^{3} \mathbb{D},{\text { Hyung Nun } \text { Kim }^{3}(\mathbb{D} \text { and Surya R. Kalidindi }}^{1,3, * \mathbb{D}}$ \\ 1 School of Materials Science and Engineering, Georgia Institute of Technology, Atlanta, GA 30332, USA; \\ zcourtright3@gatech.edu \\ 2 Marshall Space Flight Center, National Aeronautics and Space Administration, Huntsville, AL 35812, USA \\ 3 George W. Woodruff School of Mechanical Engineering, Georgia Institute of Technology, \\ Atlanta, GA 30032, USA; nicolas.leclerc@gatech.edu (N.P.L.); hnk08002@gatech.edu (H.N.K.) \\ * Correspondence: surya.kalidindi@me.gatech.edu; Tel.: +1-267-235-4208
}

Citation: Courtright, Z.S.; Leclerc, N.P.; Kim, H.N.; Kalidindi, S.R. Critical Comparison of Spherical Microindentation, Small Punch Test, and Uniaxial Tensile Testing for Selective Laser Melted Inconel 718. Appl. Sci. 2021, 11, 1061. https:// doi.org/10.3390/app11031061

Academic Editor: Alexey

S. Prosviryakov

Received: 31 December 2020

Accepted: 19 January 2021

Published: 25 January 2021

Publisher's Note: MDPI stays neutral with regard to jurisdictional claims in published maps and institutional affiliations.

Copyright: (c) 2021 by the authors. Licensee MDPI, Basel, Switzerland. This article is an open access article distributed under the terms and conditions of the Creative Commons Attribution (CC BY) license (https:// creativecommons.org/licenses/by/ $4.0 /)$.

\begin{abstract}
Standardized mechanical tests have become one of the central bottlenecks in the efficient and cost-effective exploration of the process space in advanced manufacturing processes such as additive manufacturing (AM). This paper presents a critical comparison of the relative advantages and disadvantages between emergent high-throughput mechanical test protocols, specifically, spherical microindentation and small punch test, and standardized tension tests. The critical comparison considers the effectiveness and viability of the testing protocols to rapidly screen stress-strain data and mechanical properties of candidate AM-processed metal specimens. These comparisons were performed on samples of Inconel 718 produced by selective laser melting (SLM). Modulus, yield strength, and ultimate tensile strength were evaluated, and the combination of high-throughput mechanical test protocols displayed results consistent with standard tension tests. This study shows that high-throughput mechanical test protocols can successfully produce reliable stress-strain data using significantly smaller material volume and reduced labor compared to the standardized tension tests.
\end{abstract}

Keywords: high throughput; indentation; punch test; additive manufacturing

\section{Introduction}

Metal additive manufacturing (AM) has strong potential to produce complex parts at a greatly reduced cost and time compared to conventional manufacturing techniques such as casting and forging. Attaran [1] reviewed the advantages and disadvantages of $\mathrm{AM}$ and discussed how it will impact the industry through increasing efficiency over traditional manufacturing techniques. Jared et al. [2] discussed the ability of AM to design complex structures which are difficult or impossible to manufacture using conventional manufacturing techniques. These two articles conclude that the industrial impacts, through increased design flexibility and fabrication efficiency, lead to the inevitable adoption of AM throughout many industries, from medical to aerospace. Gibson and Khorasani [3] described the growth of metal AM within the aerospace industry and discussed how companies such as General Electric have demonstrated a realization of the potential of metal AM by working to adopt metal AM techniques to greatly reduce the costs and time associated with manufacturing jet engine components. Waller et al. [4] studied nondestructive evaluation (NDE) techniques and how they can be applied to AM so AM hardware can be evaluated both in terrestrial and in microgravity environments. Their work represents NASA's vested interest in the usage of AM and it concluded with a list of recommendations for the development of NDE to enable AM.

$\mathrm{AM}$ has incredible potential to obtain novel material compositions and microstructures/chemistries that could deliver unique combinations of effective properties. Trosch et al. [5] 
determined that because of the unusual processing conditions attained in AM (e.g., extremely high cooling rates from liquid state, multiple heating cycles as material is deposited in layers), there is tremendous potential for AM to compete with forged or cast parts by producing better mechanical properties. Babuska et al. [6] focused on the study of using AM to produce intermetallic alloys with fine-tuned compositions for electromagnetic applications. These alloys would exhibit macroscopic defects if manufactured with conventional techniques, so AM allowed the production of materials which were historically inaccessible. However, attaining materials with enhanced property combinations requires systematic exploration of a very large AM process design space (each element of this space corresponds to a combination of one material chemistry and one thermomechanical history encountered in the selected AM process). Unfortunately, such explorations are limited by a lack of validated high-throughput experimental assays for rapidly evaluating the mechanical properties of AM-processed material samples.

Although the uniaxial tensile test (UTT) is standardized and commonly used throughout the manufacturing industry, it presents a major bottleneck for the AM process and materials development for several reasons. First, the high costs of sample preparation and testing preclude systematic exploration of the extremely large AM process design space mentioned earlier. Second, tensile testing requires a statistically homogeneous microstructure in the entire gauge section of the tested sample. AM samples inherently produce highly heterogeneous microstructures that may not meet the required statistical homogeneity for the standardized tensile test sample geometries. As a result, standard UTT often does not produce a consistent set of measurements for AM samples, especially in the process development stages. Kok et al. [7] reviewed an array of AM technologies and focused on the heterogeneity in microstructure and mechanical properties of AM parts. They discovered that previous research found anisotropy in the mechanical properties of AM parts and discussed how heterogeneity in microstructure led to mechanical anisotropy. The bottleneck caused by UTT slows down the innovation and implementation of metal AM components in advanced technologies. Seifi et al. [8] discusses the need for standardized methods to qualify AM materials and expresses apprehension related to the similitude between AM hardware and tensile test samples built to qualify an AM material or parameter set.

Selective laser melting (SLM) is a metal AM process that has shown incredible potential for low-cost production of specialized components for turbine and rocket engines. SLM results in near net-shape parts with minimal need for post-build machining. Gradl et al. [9] postulated that SLM is ideal for rocket engine components because it combines multiple subassemblies into a single manufacturing process, and components may be built in a fraction of the time and cost compared to conventional methods. Their work inspired the propagation of AM throughout the rocket industry which led to increased demand for development of AM process parameters for nickel-based alloys. Clinton Jr. [10] presented progress toward the development of AM for rocket engines for NASA applications and explained how cost and schedule to produce prototype engines can be drastically reduced by using AM processes such as SLM. For the aerospace industry to fully realize this potential, advances in high-throughput mechanical testing are necessary. An array of methods are being evaluated for their effectiveness in meeting the demands for low-cost and highspeed mechanical characterization. Although microtensile tests allow measurement of mechanical properties in protocols that are most similar to the standardized UTT while requiring only small amounts of material, they are largely hindered by the machining time required to produce test samples. Magagnosc et al. [11] described the use of femtosecond laser machining to combat restrictions imposed by conventional machining techniques and applied this higher efficiency machining method to the preparation of microtensile samples. While they focused on improving the machining process for microtensile samples, their work inherently indicates that the machining required to produce microtensile samples inhibits the high-throughput ability of microtensile testing. Like UTT, microtensile samples are also difficult to test in a high-throughput manner because each sample must 
be manually installed into a tensile load frame with proper alignment before it is tested. Hardness tests, using a variety of tip shapes and sizes, have long been used by the metal processing industry for quick and low-cost evaluations of the mechanical properties of their metal products. Aboulkhair et al. [12] utilized these techniques to produce maps of hardness values in an SLM-produced part to validate their hypothesis related to variations in hardness between heat-treated and as-built SLM samples. However, accurate quantitative correlations between hardness measurements and intrinsic material properties, such as tensile yield strength, that are broadly applicable to a wide variety of materials are still lacking. Rodriguez and Gutierrez [13] identified that indentation size and depth are directly related to nanohardness measurements, thereby suggesting that intrinsic material properties cannot be obtained with conventional hardness testing.

Two emergent protocols that have recently demonstrated substantial potential for rapid screening of mechanical properties are the microindentation stress-strain (MSS) protocols and small punch test (SPT) protocols. Pathak et al. [14] presented a method to determine indentation stress-strain curves from nanoindentation load-displacement data and the MSS technique used for this paper is based on their approach. Abendroth and Kuna [15] described SPT protocols which they used to determine material parameters that relate finite element (FE) simulations of SPT to experimental SPT load-displacement data. Calaf Chica et al. [16] discusses different correlation methods for predicting the ultimate tensile strength (UTS) of steel alloys from SPT load-displacement curves and asserts the accuracy of SPT for UTS determination. MSS measures indentation modulus and indentation yield strength of the material, from which one can estimate the values of Young's modulus and uniaxial yield strength for the tested sample. MSS has the capability of measuring these properties in a minimally invasive manner, while utilizing a very small volume of material. While MSS protocols share several details with standard hardness measurements, such as the ability to be performed on a metallography sample, they also exhibit many distinct differences. The MSS method presented by Pathak et al. [14] exhibited a distinct difference in that the MSS protocols utilize multiple progressively higher load cycles on the samples and utilize each unloading segment to extract one datapoint on the indentation stress-strain curve. The MSS protocols are described in greater detail in Section 2.4. Patel and Kalidindi [17] utilized FE models to establish a conversion factor of 2.2 to convert from the indentation yield strengths calculated using MSS protocols to UTT yield strength. Khosravani et al. [18] utilized the scaling factors established by Patel and Kalidindi [17] to compare nanoindentation stress-strain data to UTT for two martensitic Fe-Ni-C steels. Their findings showed that these scaling factors resulted in reasonable agreement between the nanoindentation and UTT data. Weaver et al. [19] applied experimental MSS protocols to a limited set of microstructurally graded samples of Al-6061 and identified a similar conversion factor through direct comparison with UTT. In contrast to MSS protocols, SPT protocols have been conventionally employed to estimate the plastic properties of the material such as tensile yield strength and UTS. Fernández et al. [20] demonstrated the use of SPT load-displacement data to determine UTS and yield strength of powder metal products through linear regression. They argued that the small sample size required for SPT makes it well suited for situations where a standard UTT is not possible, such as in the final sintered products made using powder metallurgy. They were successful at determining the tensile properties of three different metal powders using SPT. Abendroth [21] determined that experimental load-displacement data alone is not sufficient to determine material properties due to the non-uniform stresses experienced by the SPT sample while it is deformed to the point of failure by a spherical punch. The author suggested that FE simulations must be used in conjunction with experimental data to accurately predict material properties using SPT. SPT requires significantly less material volume and sample machining time compared to UTT. Both SPT and MSS are amenable to automated and high-throughput experimental assays.

This paper critically evaluates the viability and effectiveness of MSS and SPT protocols in rapid evaluation of mechanical properties of SLM-produced Inconel 718 samples with 
different process parameters. More specifically, the combined stress-strain results obtained using the results from both MSS and SPT protocols were directly compared with those obtained from standardized UTT protocols in terms of accuracy. This comparison directly addressed the validity of both MSS and SPT with respect to widely accepted UTT protocols and is necessary to satisfy the concerns of industrial mechanical testing experts. To accommodate the growing need for AM material and process parameter development, high fidelity mechanical testing must be done more quickly and at a lower cost. The decision to adopt MSS and SPT protocols to achieve this need rests upon the trust of mechanical testing experts in these state-of-the-art testing methods.

\section{Materials and Methods}

\subsection{Sample Prototyping Using SLM}

A total of eight tensile samples of Inconel 718 were produced at the NASA Marshall Space Flight Center, in Huntsville, AL, USA, using the SLM process. The argon atomized Micro-Melt 718 AM metal powder was provided by Carpenter Powder Products, Bridgeville, PA, USA. The SLM process involves the use of metallic powder and targeted laser patterns to generate successive layers of a part designed with computer aided design (CAD) software such as Creo View or Solid Edge.

As shown in Table 1, tensile bars 1-4 were generated using a Concept Laser M1 SLM printer while 5-8 were generated using a Concept Laser M2 SLM printer.

Table 1. Details of the Inconel 718 SLM samples produced for this study.

\begin{tabular}{ccc}
\hline Sample Number & HIP $(\mathbf{Y} / \mathbf{N})$ & Machine \\
\hline 1 & $\mathrm{~N}$ & M1 \\
2 & $\mathrm{~N}$ & M1 \\
3 & $\mathrm{~N}$ & M1 \\
4 & $\mathrm{Y}$ & M1 \\
5 & $\mathrm{~N}$ & M2 \\
6 & $\mathrm{~N}$ & M2 \\
7 & $\mathrm{~N}$ & M2 \\
8 & $\mathrm{Y}$ & M2 \\
\hline
\end{tabular}

M1 and M2 are both powder bed SLM printers, with the primary difference being that the M2 was designed with built-in gloves to allow manipulation of the as-built part while maintaining the inert build environment. All eight tensile bars were printed vertically as cylinders with a diameter of $12.7 \mathrm{~mm}$. Samples 1-8 were all printed using the same SLM process parameters and they all underwent a stress relief after being removed from the SLM build plate. Samples 4 and 8 underwent additional post-build heat treatment using hot isostatic press (HIP) to further increase their density, with the goal of increasing their intrinsic mechanical properties through the reduction in microstructural porosity. This sample set was designed to capture the natural variance in SLM processing for one selected material composition, which can produce a corresponding variance in the mechanical properties.

\subsection{Uniaxial Tensile Test (UTT)}

ASTM-E8 [22] was the standard used to machine UTT samples. It requires a gauge diameter of $6.35 \mathrm{~mm}$ and a length of $7.62 \mathrm{~cm}$. Following machining, samples were placed into a screw-driven mechanical actuator with an MTS $6.6120 \times 10^{-1}$ load cell with a $\pm 22,000$ pound force capacity and an Instron 8862 linear variable differential transformer with an operating range of $\pm 5.08 \mathrm{~mm}$. UTT was performed to failure on all eight SLM-built tensile bars. A displacement rate of $5.08 \mathrm{~mm} / \mathrm{min}$ was maintained until $2 \%$ strain was reached, after which the rate was increased to $12.7 \mathrm{~mm} / \mathrm{min}$. Strain data was obtained using a $2.54 \mathrm{~cm}$ extensometer calibrated to $50 \%$ strain. The MTS load cell obtained load data, which was subsequently converted to stress using the measured sample geometry. 
The stress-strain curves resulting from these tests were analyzed to determine modulus, yield strength, and UTS. ASTM-E8 [22] is a mechanical testing standard which is widely accepted throughout the aerospace industry and all of the UTT reported in this study were performed in accordance with ASTM E8.

\subsection{Sample Preparation for MSS and SPT}

Following tensile testing, the ends of the broken bars were used to extract MSS and SPT samples as shown by the cut plan in Figure 1.

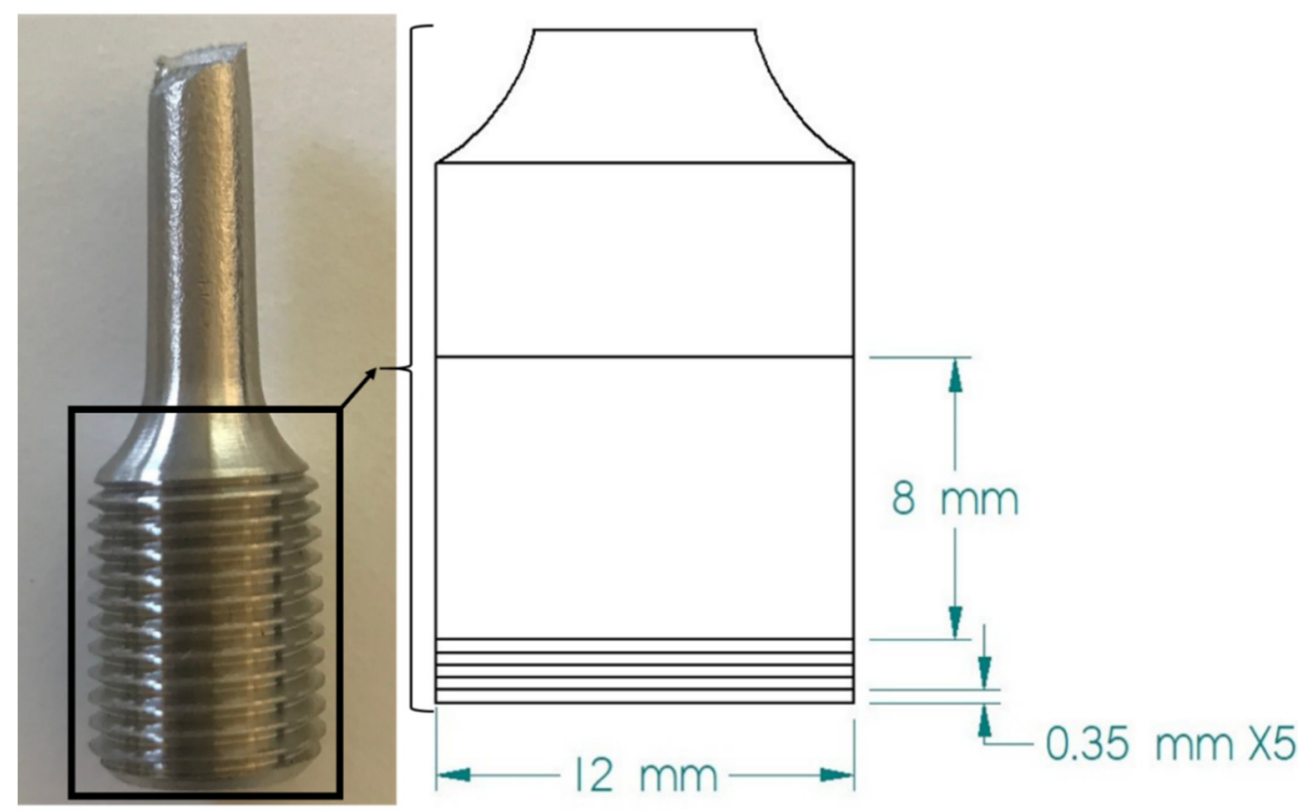

Figure 1. The cut plan used to extract small punch test (SPT) and microindentation stress-strain (MSS) samples from the ends of the tensile bars after uniaxial tensile test (UTT).

An $8 \mathrm{~mm}$ thick sample was extracted for MSS and five $0.35 \mathrm{~mm}$ thick samples were extracted for SPT. Excision of MSS and SPT samples from the ends of the tensile bars allows for a high-fidelity correlation between the MSS, SPT, and UTT stress-strain results. Given the differences in the cross-sections of the gauge section and the end section of the tensile bars, it was reasonable to assume that the end sections have not been exposed to any plastic deformation during UTT. The MSS and SPT samples were sliced with wire electrical discharge machining (WEDM) to ensure that the top and bottom surfaces remained roughly parallel. WEDM was performed at low heat and travel speed to result in a minimal thickness recast layer. The thickness of the WEDM recast layer was measured to be approximately $15 \mu \mathrm{m}$ from an optical image taken on the cross-section into the sample thickness. The recast layer was removed during subsequent polishing.

\subsection{Microindentation Stress-Strain (MSS) Protocols}

Samples for MSS were prepared using standard metallography protocols. Each machined MSS sample was mounted using a cold mounting epoxy process. The cold mounting epoxy process involved mixing 4 parts Buehler EpoxiCure 2 Epoxy Resin with 1 part Buehler EpoxyCure 2 Epoxy Hardener. Water lubricated grinding was initiated with 800grit sandpaper and progressed through 1000-, 1200-, 2400-, and 4000-grit sandpapers prior to polishing. After grinding, the samples were subsequently polished on a $3 \mu \mathrm{m}$ and $1 \mu \mathrm{m}$ polishing pad using appropriate diamond pastes and alcohol-based lubricant. Next, a polishing pad with $0.04 \mu \mathrm{m}$ colloidal silica polishing suspension was applied. Polishing was completed by vibropolishing the samples for $24 \mathrm{~h}$ using a colloidal silica suspension at 30 RPM. Between each polishing step, the samples were rinsed with soapy water and ethanol to remove polishing remnants from the previous step. 
MSS testing was carried out using a Zwick-Roell ZHU2.5 Zwick Hardness Tester. A Brinell spherical indenter tip with a $6.35 \mathrm{~mm}$ radius was utilized and produced a residual indentation radius of approximately $180 \mu \mathrm{m}$ depending on the sample. Sample grain sizes varied between $30 \mu \mathrm{m}$ and $50 \mu \mathrm{m}$, so the residual indent covered no less than 10 grains. An array of 6 to 12 indentations was performed while maintaining a $2 \mathrm{~mm}$ distance between the indentation center points and from the edge of the sample. This ensured a center-to-center distance between indentations of approximately 10 times the residual indent radius; this exceeded the separation requirements described in ASTM-E10-12 to prevent interactions between the indentations or with the edge of the sample. ASTM-E10-12 [23] states that a minimum center-to-center spacing of six times the residual indentation diameter shall be used. Weaver et al. [19] followed this criterion for the separation distance between indents to produce results from their indentation experiments performed on aluminum alloys that were consistent with tensile stress-strain data. The averaged measured response from the multiple MSS tests on a sample are assumed to reflect the bulk response of the material.

Figure 2 depicts schematically the indenter-sample geometry under load and provides a visual representation of variables discussed in the following paragraphs.

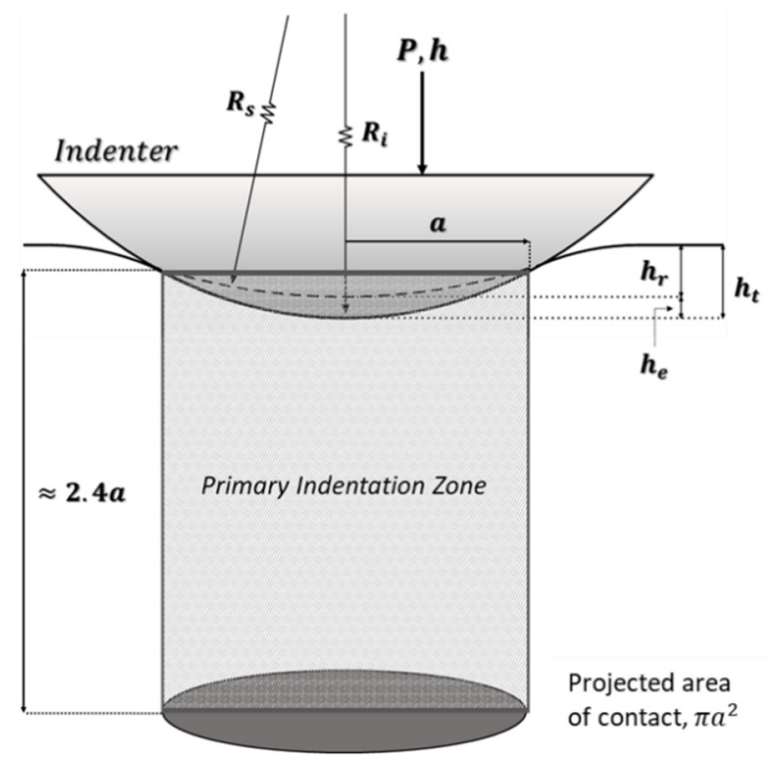

Figure 2. Indentation schematic showing the variables involved in indentation stress-strain protocols. The indentation stress definition is based on the load applied over the projected area of contact, $\pi a^{2}$, where $a$ is the contact radius. The indentation strain definition is based on the dimensions of the primary indentation zone, which describes a cylindrical domain of height $2.4 a$, and radius $a$ being compressed by the total indenter displacement, $h_{t}$ which is the sum of the sample elastic displacement, $h_{e}$, and the residual displacement, $h_{r}$.

A typical measured load-displacement curve produced using the MSS protocols is shown in Figure $3 a$. 

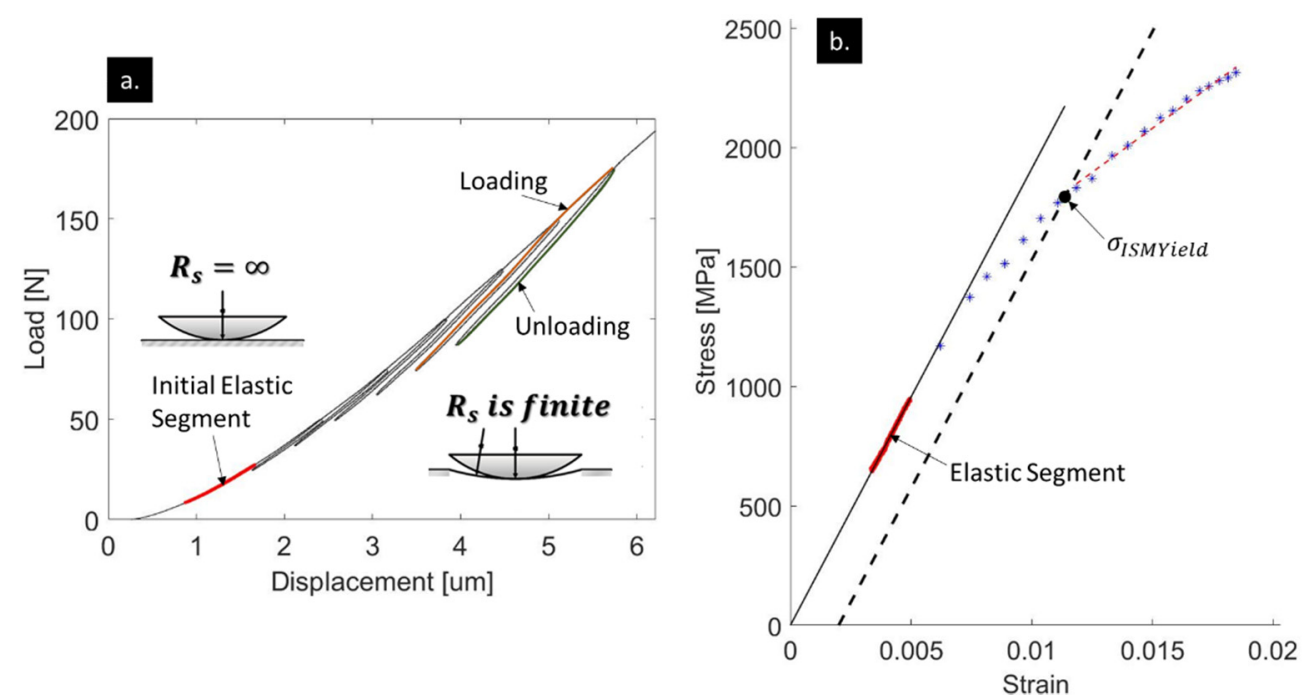

Figure 3. (a) A typical measured load-displacement response in the indentation protocols used in this work. The initial elastic response shown is analyzed using Hertz's theory. (b) The corresponding indentation stress-strain curve extracted from (a).

As mentioned earlier, each unloading segment (indicated in Figure 3a) was analyzed to produce one datapoint on the indentation stress-strain curve. Only a small number of unloading segments are shown in Figure 3a for clarity. In the results presented in this work, $\sim 20-25$ unloading segments were utilized in the extraction of each indentation stressstrain curve. The elastic loading and all of the unloading segments were subsequently analyzed using Hertz's theory for frictionless elastic contact between two quadratic surfaces. Pathak et al. [14] demonstrated and validated this method by testing both aluminum and tungsten samples and directly comparing the results to those obtained using a continuous stiffness measurement. The following relationships are utilized:

$$
\begin{gathered}
P=\frac{4}{3} E_{e f f} R_{e f f}^{\frac{1}{2}} h_{e}^{\frac{3}{2}} \\
a=\sqrt{R_{e f f} h_{e}}
\end{gathered}
$$

where $P$ and $h_{e}$ are the indentation load and the elastic indentation displacement, respectively, and $a$ is the contact radius (see Figure 2). $E_{\text {eff }}$ and $R_{e f f}$ are the effective indentation modulus and the effective radius of the indenter-specimen system, and are defined as

$$
\begin{gathered}
\frac{1}{E_{e f f}}=\frac{1-v_{s}^{2}}{E_{s}}+\frac{1-v_{i}^{2}}{E_{i}} \\
\frac{1}{R_{e f f}}=\frac{1}{R_{i}}+\frac{1}{R_{s}}
\end{gathered}
$$

where $E, R$, and $v$ are Young's modulus, radius, and Poisson's ratio, respectively, and the subscripts $i$ and $s$ denote indenter and specimen, respectively.

In the MSS protocols, one employs Hertz's theory described above to each unloading segment to estimate the contact radius needed to compute the indentation stress and the indentation strain corresponding to the start of the unloading segment (see Figure 3a). Kalidindi and Pathak [24] reformulated Hertz's theory (Equations (1) and (2)) to derive the definitions of indentation stress and strain used in MSS protocols as

$$
\sigma_{\text {ind }}=\frac{P}{\pi a^{2}}
$$




$$
\varepsilon_{\text {ind }}=\frac{4}{3 \pi} \frac{h}{a}
$$

Donohue et al. [25] validated the above definitions for indentation stress and strain in their indentation simulations by observing indentation responses in the context of stress and strain fields. The indentation analysis described above produces a linear relationship in the initial elastic loading and exhibits a smooth transition to a nonlinear elastic-plastic response.

MSS protocols start with the analyses of the initial elastic segment (see Figure 3a). One of the main challenges in this analysis is the zero-point correction to correct for various unavoidable factors such as imperfect initial contact, sample surface irregularities, imperfect indenter shape, etc. This task is accomplished by fitting the initial elastic segment to the following expression derived from Hertz's theory expressed above:

$$
\begin{gathered}
\left(\widetilde{h}-h^{*}\right)=k\left(\widetilde{P}-P^{*}\right)^{\frac{2}{3}} \\
k=\left[\frac{3}{4} \frac{1}{E_{e f f}} \frac{1}{\sqrt{R_{e f f}}}\right]^{\frac{2}{3}}
\end{gathered}
$$

where $\widetilde{P}$ and $\widetilde{h}$ denote the measured load and displacement, respectively, and $P^{*}$ and $h^{*}$ denote the zero-point corrections for the load and displacement, respectively. Note that for the initial elastic loading $R_{e f f}=R_{i}$ and all of the indentation displacement is elastic. Therefore, the regression analyses of the initial loading segment will also provide an estimate of $E_{e f f}$, which is assumed to remain the same even after the initiation of plastic deformation in the indentation experiment. Pathak and Kalidindi [26] observed that because the averaged plastic strain experienced under the spherical indenter is relatively low, there were no significant changes to the effective indentation modulus of the sample material in the primary indentation zone (see Figure 2). The $P^{*}$ and $h^{*}$ corrections identified in this analysis are applied to the entirety of the measured load-displacement curve.

Next, each unloading segment after the initiation of plastic deformation in the indentation experiment is analyzed to produce a single data point on the indentation stress-strain curve (see Figure $3 \mathrm{~b}$ ). This is accomplished by fitting the unloading segment to the following expression derived from Hertz's theory described above:

$$
h_{t}=k P^{\frac{2}{3}}+h_{r}
$$

where $h_{t}$ and $h_{r}$ are the total indentation displacement and the residual displacement after the disengagement of the indenter (see Figure 2), respectively. Although $k$, used in Equation (9), is defined by Equation (8), it should be noted that $R_{e f f}$ is no longer a known quantity because $R_{s}$ has changed with the initiation of plastic deformation in the indentation measurement. However, regression of each unloading segment to Equation (9) can be used to estimate the value of $k$ and $h_{r}$, which can then be used to estimate $R_{e f f}$ using Equation (8) and compute $h_{e}=h_{t}-h_{r}$. The contact radius $a$ can then be estimated using Equation (2). These estimates now allow the estimation of indentation stress and indentation strain values at the start of each unloading segment using Equations (5) and (6). From the aggregated indentation stress-strain points (see Figure $3 b$ ), a $0.2 \%$ offset yield criteria is determined as the indentation yield strength. Weaver et al. [19] demonstrated in their indentation experiments that using a $0.2 \%$ offset yield produced reliable results that were comparable to tensile test data on their aluminum alloys.

\subsection{Small Punch Test (SPT) Protocols}

Abendroth and Soltysiak [27] performed a review of the historical applications of SPT for mechanical evaluation of materials and indicated that comparing experimental SPT results with FE computations is necessary to gather relevant material properties. Early 
development of SPT was performed to satisfy niche applications in the nuclear industry. Manahan et al. [28] suggested that a low cost and quick test was necessary to assess mechanical behavior in a variety of alloys used in nuclear reactors. Mao et al. [29] further elaborated on the application of SPT in the nuclear industry by arguing that SPT can be used to study mechanical degradation in neutron-irradiated materials using exceedingly small material volumes. García et al. [30] used a test method where a thin plate ( 0.5 mm thickness) was firmly clamped between two dies with a circular opening centered over the plate in both the upper and lower dies (see Figure 4a). The method they used was found to produce consistent load-displacement results and was employed for this research study. The load-displacement results from each SPT sample were utilized in this research study to obtain a true stress-true plastic strain response from which UTS and yield strength were determined. Standards are currently being developed for SPT. CWA-15627 [31] was an initial attempt at standardization of SPT which took place at the CEN Workshop Agreement-CWA in Europe. Bruchhausen et al. [32] more recently attempted to document and standardize SPT by summarizing the current practice of SPT and the details for a future standard.
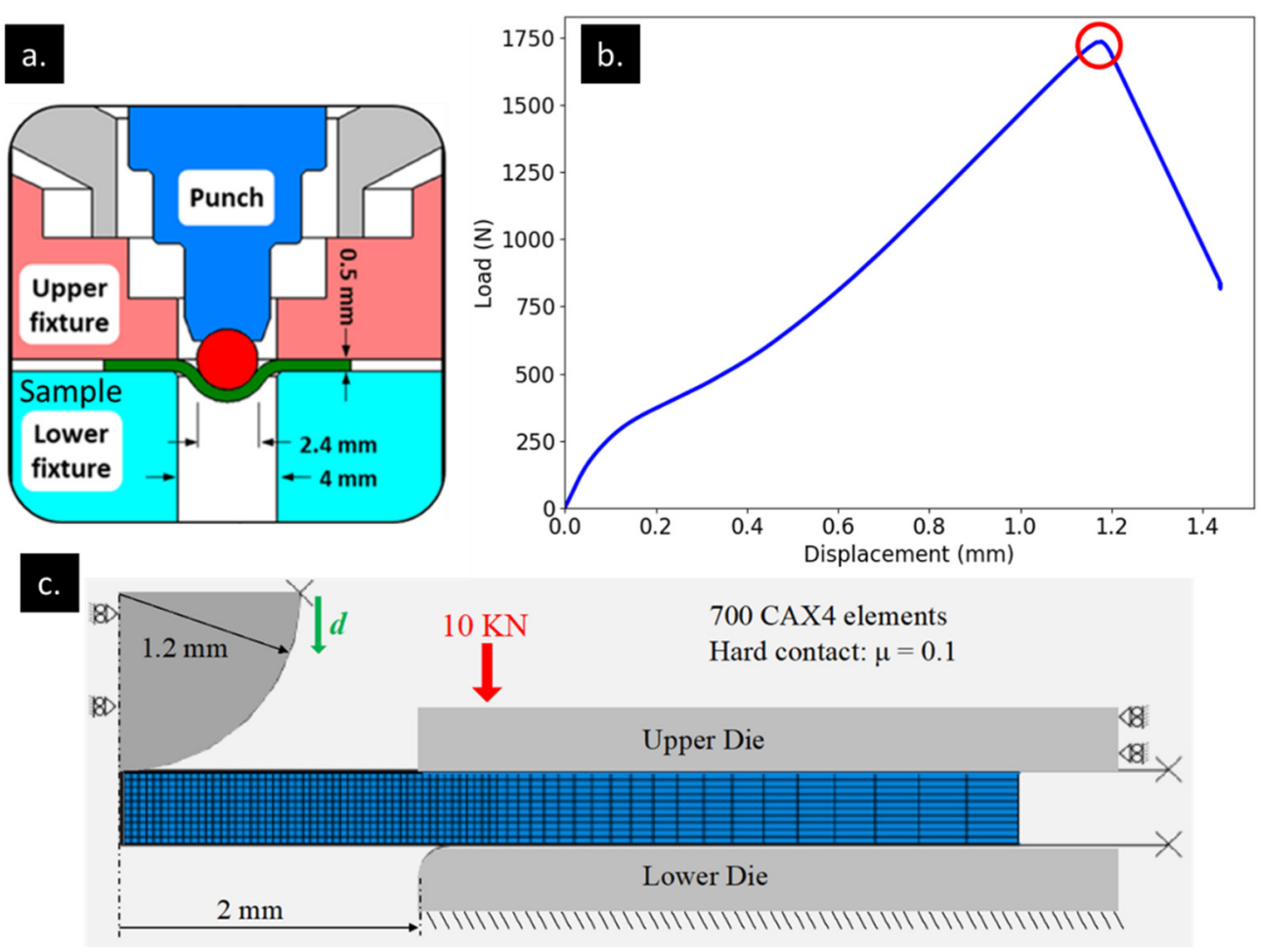

Figure 4. (a) A schematic of the SPT experimental setup. (b) A typical measured SPT loaddisplacement curve. (c) Finite element (FE) mesh used for SPT simulations.

SPT samples were prepared for testing by directly polishing both sides of the samples against an 800-grit followed by a 1000-grit sandpaper until the removal of the as-WEDM recast layer is visually indicated. After polishing, each SPT sample was measured for thickness in three locations using a micrometer and variance within each sample was maintained below $15 \mu \mathrm{m}$. An average of the thickness measurements was recorded and used in the simulations and analysis necessary to extract intrinsic mechanical properties from SPT experimental results. SPT was carried out using the same indention machine that performed MSS. This process involved placing an SPT sample over a $4 \mathrm{~mm}$ hole in a lower fixture and clamping the sample with an upper fixture as shown in Figure 4a. A lubricant was applied to the surface of the SPT sample to reduce friction during the contact with the punch. García et al. [30] demonstrated successful SPT repeatability by using a $2.4 \mathrm{~mm}$ punch tip and a $4 \mathrm{~mm}$ fixture hole, so those dimensions were used in the 
application of this work. The assembled fixture containing the clamped sample was then placed on the indenter stage. The optical microscope on the indenter machine was used to adjust the placement of the sample fixture so that the indenter was at the center of the unsupported span of the sample (see the schematic in Figure 4a). Leclerc et al. [33] used a punch displacement rate of $0.5 \mathrm{~mm} / \mathrm{min}$ and successfully demonstrated the accuracy of test results using this rate on a variety of different materials. As such, this study used an identical loading rate during SPT analysis. The test was terminated when the punch force exhibited a clear peak, as shown in the circle in Figure $4 \mathrm{~b}$, which indicated that the sample has been punched through.

A few different corrections to the measured load-displacement curves were necessary before applying SPT analyses protocols described in Section 3.2. A zero-point correction in the form of the subtraction of a constant value was needed for the measured displacement. This constant value is established by fitting the very early elastic portion of the SPT loaddisplacement curve to a linear model and examining where it intersects the x-axis of the plot. This correction ensures that the initial portion of the measured load-displacement curve goes through the $(0,0)$ point. Leclerc et al. [33] determined that a second correction is needed to account for the machine compliance and other similar effects. This correction function (i.e., discrepancy between displacement in the machine loading assembly outside of the sample and FE model displacement) is established as a function of the applied load by performing SPT on a suite of different material samples with known uniaxial stress-strain responses. The machine compliance is then standardized and applied on all SPT measurements made on the same machine. In other words, the displacement in the machine corresponding to the imposed load in the SPT load-displacement is estimated, and applied as a subtractive correction to the measured total displacement in the SPT. This subtractive correction also accounts for any other sources of disagreement between the experiments and FE simulations (e.g., small differences in geometry of the sample or the dies or simplifications made in the FE models).

\section{Results}

\subsection{MSS Measurements}

Multiple (>5) MSS measurements were conducted on each of the eight SLM samples, with approximately 20 unloading segments in each test. Recall that each unloading produces one data point on the indentation stress-strain curve. Therefore, a total of roughly 20 data points on the indentation stress-strain curve were obtained for each SLM sample.

Patel and Kalidindi [17] explored and validated scaling factors used to convert MSS stress-strain curves to UTT stress-strain curves have been established in their prior work using a combination of FE models and experiments. The authors of this work demonstrated that these scaling factors account for the inherent heterogeneity of the deformation induced in the indentation experiments as well as the differences in the stress (and strain) states compared to the uniaxial tests. In this same work, they established equations which can be used to convert indentation stress-strain curves to uniaxial stress-strain curves, and they are summarized below:

$$
\begin{gathered}
\varepsilon_{e M S S}=\frac{\sigma_{M S S}}{E_{e f f}} \\
\varepsilon_{p_{M S S}}=\varepsilon_{T M S S}-\varepsilon_{e M S S} \\
\varepsilon_{T U T T}=\left(\frac{\varepsilon_{e M S S}}{2}\right)+\left(\frac{\varepsilon_{p_{M S S}}}{1.3}\right) \\
\sigma_{U T T}=\frac{\sigma_{M S S}}{2.2}
\end{gathered}
$$

where $\varepsilon_{e M S S}$ is the elastic strain from MSS analysis, $\varepsilon_{p_{M S S}}$ is the plastic strain from MSS analysis, $\varepsilon_{T M S S}$ is the total strain from MSS analysis, $\sigma_{M S S}$ is the stress from MSS analysis, $E_{\text {eff }}$ is the indentation modulus of the sample, $\varepsilon_{T U T T}$ is the total strain in UTT, and $\sigma_{U T T}$ is the stress in UTT. In the linear elastic portion of the UTT stress-strain curve, only the first 
term in Equation (9) is used (i.e., the plastic strain in this regime is assumed to be zero). A comparison of the UTT stress-strain points estimated from MSS measurements using Equations (10)-(13) is compared with the direct measurement of UTT stress-strain curves for Sample number 8 in Figure 5.

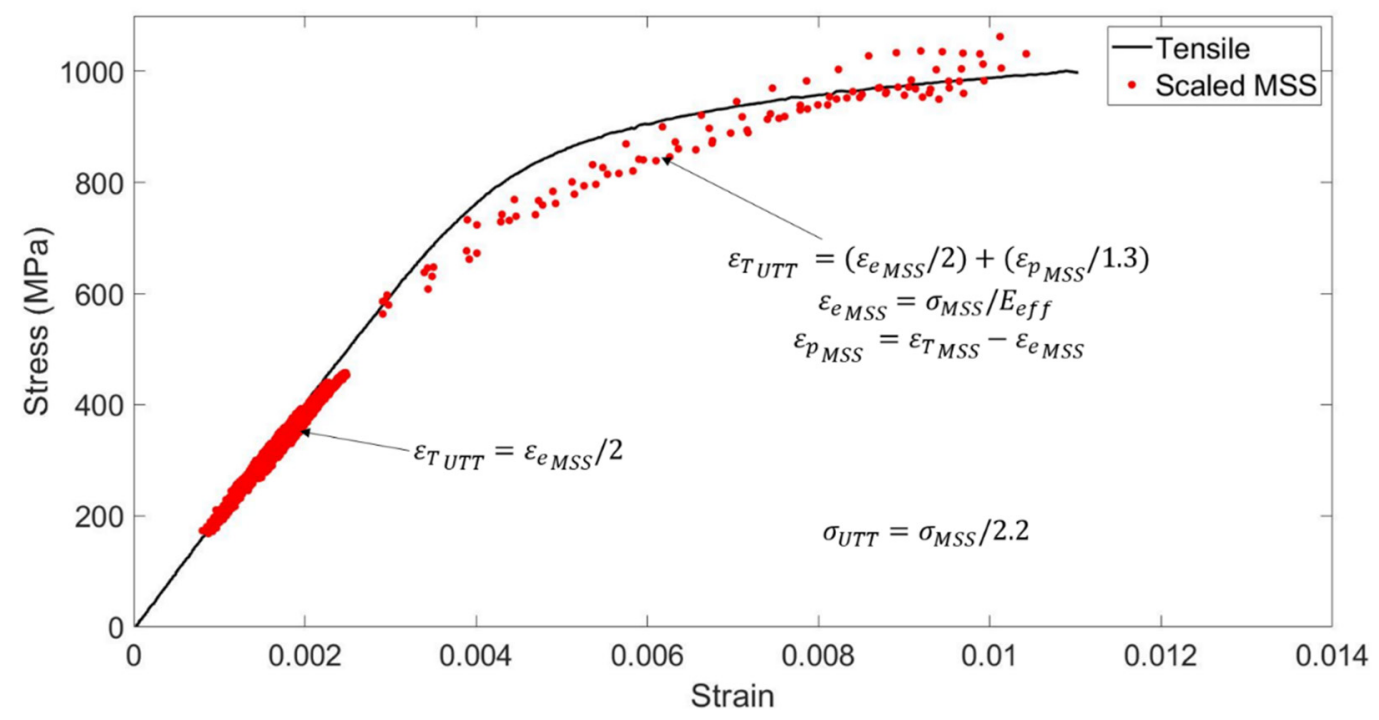

Figure 5. An overlay of data collected from five MSS tests conducted on Sample number 8 on the corresponding UTT stress-strain curve.

It is seen that the data points extracted from MSS measurements are in good agreement with large portions of the measurements obtained directly from UTT protocols. The highest discrepancy between these curves was seen in the elastic-plastic transition. These observations are consistent with results presented by Patel and Kalidindi [17] in their indentation simulations, and the authors have concluded that these differences are likely a consequence of the differences in the elastic-plastic transition in the MSS and UTT test methods. Due to the differences in geometry between UTT and MSS, the transition from elastic deformation to constant hardening behavior in the material occurs at a different rate. In UTT, load is applied over a constant cross-sectional area, so there is a bilinear stress-strain response where the first linear segment represents the elastic response of the material while the second linear segment represents the plastic response of the material where strain hardening is constant. In MSS, the elastic-plastic transition region is nonlinear because the contact radius between the indenter and sample is continuously changing as load increases.

Khosravani et al. [18] have demonstrated that the values of Young's moduli of the samples can be estimated using Equation (3) and the MSS measurements of $E_{e f f}$ obtained from the initial elastic portions of the indentation stress-strain curves. These estimated values for the Inconel 718 samples in this study are compared against their corresponding direct measurements from UTT in Figure 6.

For seven of the eight samples, the discrepancy between the MSS and UTT measurements was $\leq 8 \%$. Sample number 4 exhibited a higher difference in the modulus values between these two tests and is considered to be an outlier. The reasons for this discrepancy are not yet clear, but may reflect a higher level of heterogeneity or anisotropy in this AM sample. Kok et al. [7] reviewed literature on a wide range of metal AM processes and focused on how the heterogeneity and anisotropy in an AM microstructure can lead to variations in mechanical properties. Since the material volume probed in the MSS is significantly smaller compared to UTT, heterogeneity in the sample is likely to result in some of the differences noted in the modulus values in Figure 6. Moussa et al. [34] studied the issue of heterogeneity in spherical indentation load-displacement responses and how to develop a confidence domain using the variation between experimental load-displacement 
curves. They focused on the error distribution between FE simulations and experimental load-displacement curves and on the average representative strain from multiple experimental tests to determine a confidence domain. It should also be noted that the Equation (3) is based on an isotropic material response. Consequently, any anisotropy in the material response can also contribute to the observed differences in Figure 6.

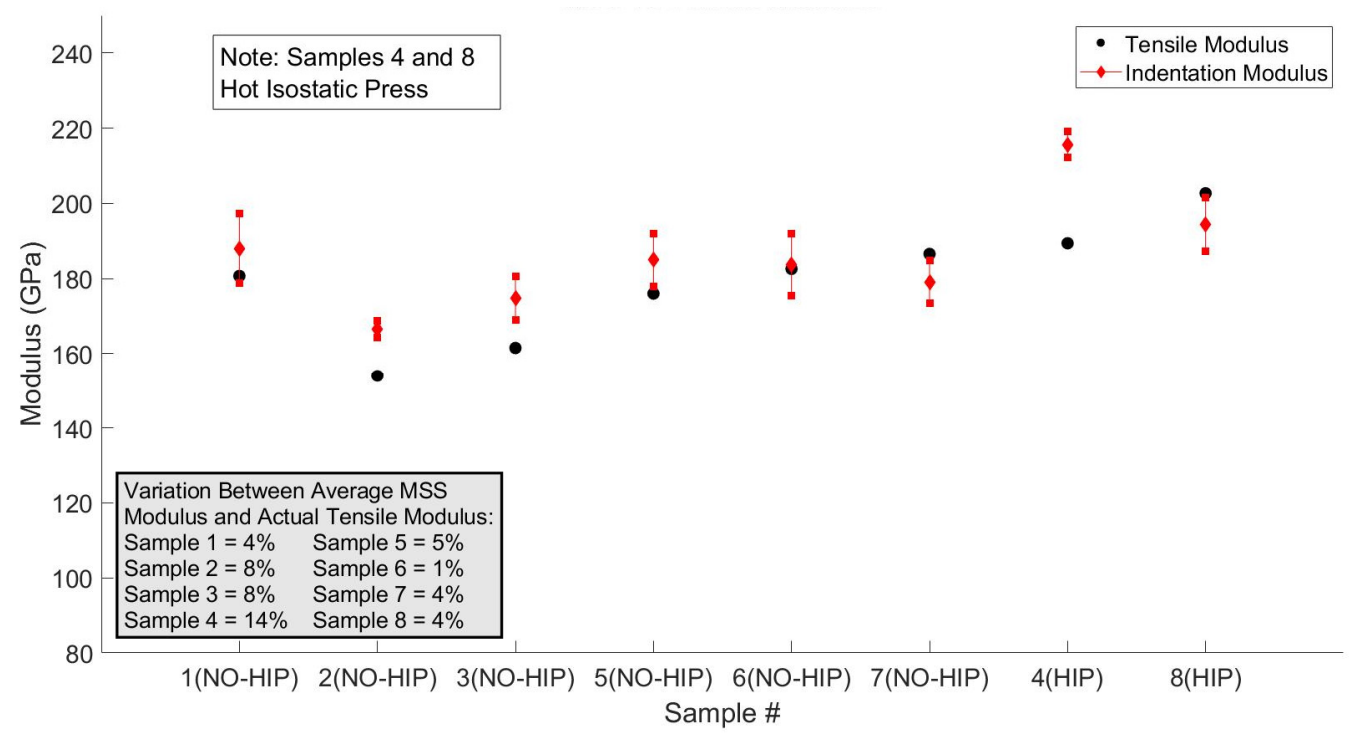

Figure 6. Estimated values of Young's modulus obtained from MSS tests presented as averages and standard deviations, along with the corresponding UTT measurements. The table summarizes the percent variation between the average of the MSS moduli and the UTT modulus for each sample.

The $0.2 \%$ offset UTT yield strength can be estimated from the $0.2 \%$ offset MSS yield strength by dividing it by a scaling factor of 2.0 as Patel and Kalidindi [17] demonstrated and validated in their FE simulation studies. Figure 7 shows a comparison of the average and standard deviation of the tensile yield strength values extracted from MSS measurements for all samples tested in this work against the yield strength values measured directly from the UTT.

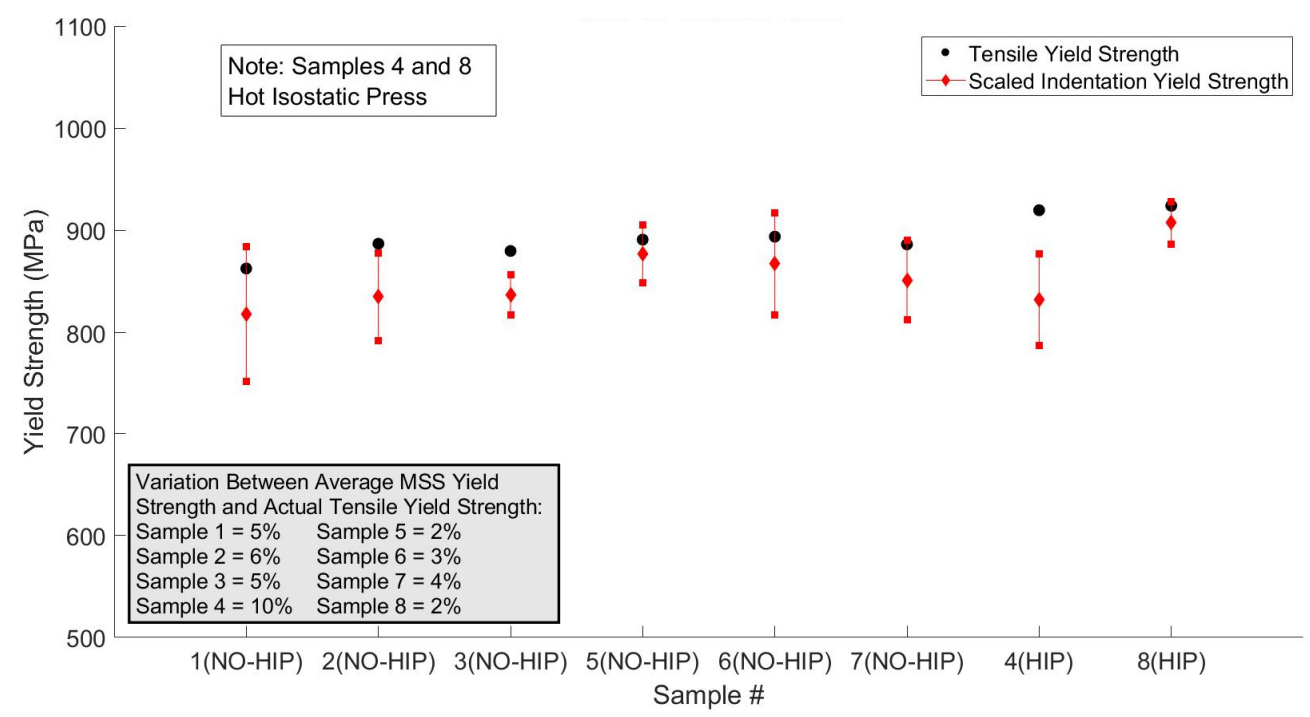

Figure 7. Estimates of yield strengths from MSS tests presented as averages and standard deviations, along with the corresponding UTT measurements. The table summarizes the percent variation between the average MSS estimated yield strength and the UTT measured yield strength. 
It is seen that the MSS estimated values are in particularly good agreement with the values measured directly from UTT. In fact, the largest discrepancy in the MSS estimated yield strengths among the eight samples was $10 \%$, and seven samples exhibited an error $\leq 6 \%$. These comparisons demonstrate the high reliability of MSS protocols in determining the yield properties of an AM material. The highest difference of $10 \%$ was observed in Sample number 4 . Given the role of elastic modulus in the estimation of the contact radius, indentation stress, and indentation strain values, it is not surprising that the sample that produced an erroneous estimate of the modulus also produced the highest error in the estimate of yield strength. It was also observed that the scaling factor of 2.0 resulted in a consistent underprediction of yield strength. Weaver et al. [19] observed a similar trend in their experimental results on aluminum alloys, where they concluded that a factor of 1.89 resulted in higher accuracy estimates. It is reiterated that the scaling factor of 2.0, used in the present study, was determined based on FE simulations that employed isotropic plasticity material constitutive laws. Therefore, this difference could indicate some degree of anisotropy in the mechanical response of the AM sample. As already described earlier, the heterogeneity of the microstructure in the AM sample at the scale of the indentation zone could also contribute to the observed differences.

\subsection{SPT}

Recently, new analysis protocols have been demonstrated for extracting the uniaxial (tension or compression) stress-strain curves from the SPT measured load-displacement curves. Leclerc et al. [33] utilized a FE library approach to predict the parameters of the Swift hardening law which were used to extract uniaxial stress-strain curves from SPT experimental data. Abendroth and Kuna [15] used a neural network trained on FE simulations to extract 5 out of 12 parameters of the Gurson-Tweergard-Needleman model and their approach was referenced in Leclerc's work. The protocols employed for this research utilized an FE model (see Figure 4c) to connect the material's uniaxial stress-strain material response (provided as an input to the FE model) and the SPT load-displacement curve (obtained as an output from the FE model). The FE model was comprised of 700 elements which were biased towards the center of the sample as seen in Figure 4c. Through a dictionary approach, the uniaxial stress-strain response input to the FE model was systematically revised until the FE-predicted SPT load-displacement curve matched well with the measured SPT load-displacement curve. Chen et al. [35] applied a similar approach to successfully determine the orientation of grains using electron backscatter diffraction images. Yabansu et al. [36] also used a dictionary approach to reconstruct microstructure images by means of spatial statistics of a microstructure.

The procedure described above has been applied in this work to recover uniaxial stress-strain responses of the samples from their SPT load-displacement measurements. Kleemola and Nieminen [37] investigated the use of a variety of strain hardening laws, including Swift law, for their applicability to describing stress-strain curves. In this work, approximately 2500 simulations were performed using Abaqus CAE for each sample thickness, with each simulation using a different set of plasticity parameters defined by the Swift law as

$$
\sigma=K\left(\varepsilon_{0}+\varepsilon_{P}\right)^{n}
$$

where $K$ is the strength coefficient, $\varepsilon_{0}$ is the prior plastic strain value, $\varepsilon_{P}$ is the true plastic strain, $n$ is the strain hardening exponent, and $\sigma$ is the true stress. In the formalism of Swift law in Equation (14), $K, \varepsilon_{0}$, and $n$, serve as the plasticity parameters. The FE-predicted load-displacement curves of a large library of simulations, that covers a broad range of possible plasticity parameters, were compared to the measured load-displacement curve using an error measure defined as the mean absolute differences between the two curves computed at uniform intervals on the displacement axis. The best three curves (with the lowest errors with respect to the experimental curve) are shown in Figure 8a along with a direct measurement for Sample number 4. These three curves were essentially 
indistinguishable and the use of the three best simulation curves represents the negligible variation between the best-matching simulation curves.
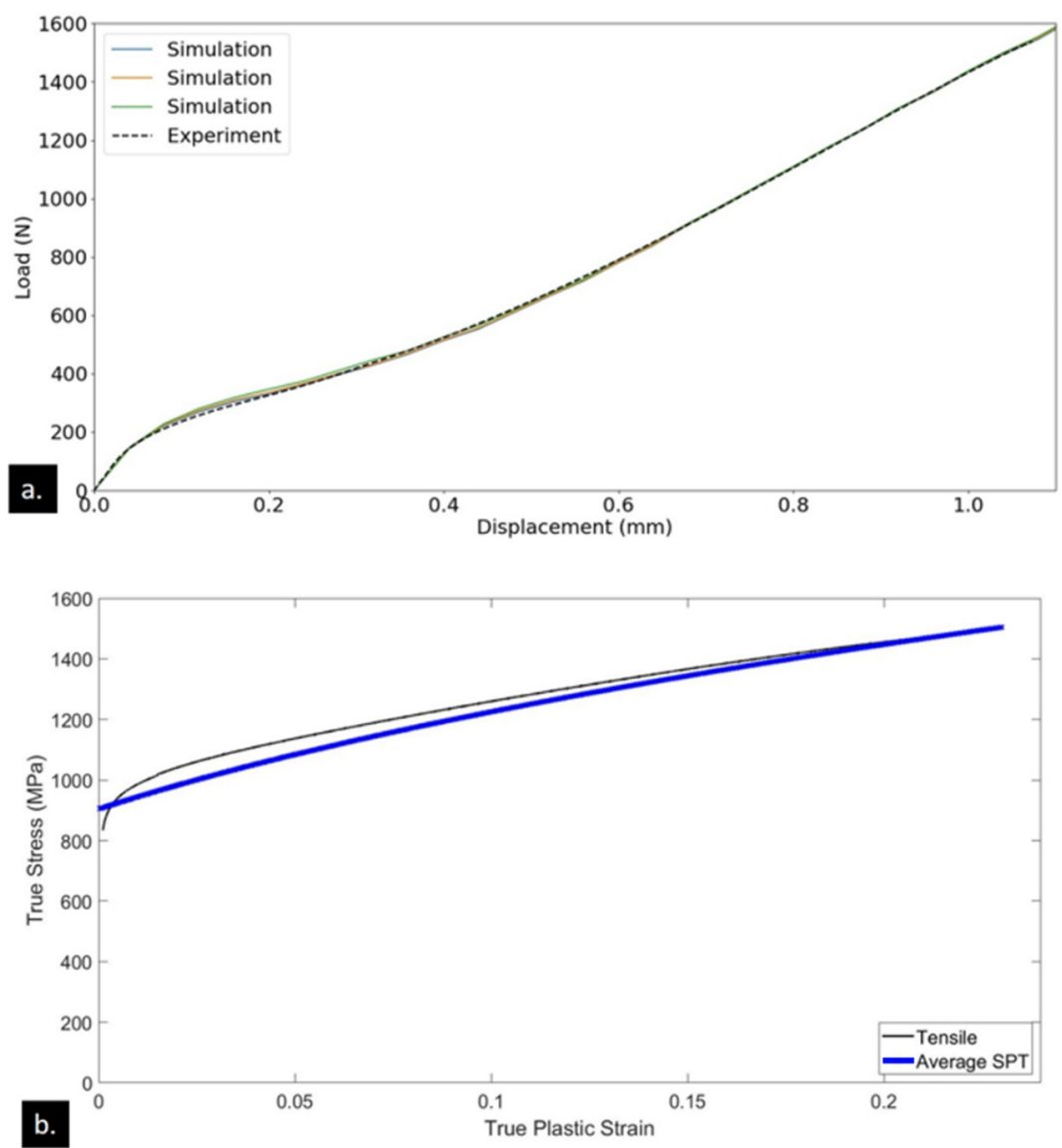

Figure 8. (a) Comparison of the measured SPT load-displacement curve on Sample number 6 with the three closest FE-predicted curves (i.e., exhibiting the lowest mean absolute error with respect to the measurement). (b) The stress-strain curve corresponding to the average $K, \varepsilon_{0}$, and $n$ values of the best fit solutions in (a) compared against the corresponding UTT response for the same sample.

An average of the $K, \varepsilon_{0}$, and $n$ values for the best-fit curves was taken to produce the estimated uniaxial stress-strain response (see Figure $8 b$ ) from the SPT load-displacement measurement. Clearly, the stress-plastic strain curves recovered from the SPT measurements were in very good agreement with the one measured in standard UTT protocols. Similar agreements were obtained for (no less than 3) SPTs on each of the eight samples tested in this work.

\section{Discussion}

\subsection{MSS}

MSS has a clear advantage with its ability to determine Young's modulus and yield strength of a given sample in a minimally invasive manner. Zarzour et al. [38] demonstrated indentation techniques as a powerful tool for extracting location-specific properties on the weld heat affected zones of manufactured final products. Kim et al. [39] further demonstrated the desirability of indentation techniques by testing polymer matrix composite-based biomedical implant prototypes where standard mechanical test methods were difficult due to a lack of material volume at the location of interest. These authors have demonstrated in their respective work the potential utility of indentation-based techniques to be easily adapted for quality control/assurance applications for the manufactured final product both during its initial deployment as well as its in-service lifetime. This is enabled by the small material volumes probed by MSS. 
Since SLM and other AM techniques intentionally employ varied process parameters for thin- and thick-walled regions of an AM build, it becomes important to evaluate and certify location-specific properties in the final AM-produced product. Clijsters et al. [40] discussed the variations in melt pool thermal characteristics inherent to geometry changes in an SLM build. They suggested parameter variations between thin- and thick-walled regions of a build. MSS can satisfy the need to understand mechanical property variation throughout an AM build by producing a map of test results along a cross-section of a part. Aboulkhair et al. [12] verified indentation testing as a way to map property variations between as-built and heat treated SLM samples. As performed in this work, an AM part may be sectioned with WEDM and polished before MSS is performed in a fully automated fashion. Figure 7 shows how MSS was found to track UTT results using only a small material volume and represents the validity of MSS for determining yield strength in SLM Inconel 718.

MSS is ideally suited for rapid screening of large materials' chemistry and process design spaces using high-throughput assays that can potentially be automated. This becomes especially attractive when MSS is combined with a suitable strategy for highthroughput prototyping of sample libraries with different chemical compositions and/or process histories. Czotscher et al. [41] researched laser deep alloying as a method to produce new construction materials in a high-throughput manner and coupled high-throughput sample production with indentation materials characterization. Tsai and Flores [42] utilized laser deposition to generate compositionally graded metallic glass samples and extracted modulus values from nanoindentations so they could find trends between composition and modulus. Both of these studies demonstrate how high-throughput material production can be coupled with high-throughput indentation testing to rapidly characterize new materials. Parvinian et al. [43], in their work using MSS protocols, has presented dramatic reductions in costs associated with mechanical property assessments which traditionally use UTT and espoused that this cost may be further reduced with more efficient data collection and analysis. The MSS performed in this study demonstrated similar cost reductions to those found by Parvinian and allowed for increased data collection from AM materials where rapid material qualification is necessary to meet demand.

MSS is also well suited for situations where full automation is required, such as inspace manufacturing applications. Coll et al. [44] discusses NASA plans to automate the process of refueling a satellite in space using advanced robotics. Similar robotic technologies may be used on an AM build created in space to automatically perform both polishing and indentation tests for materials' characterization. This process will require far less complexity than a UTT setup which would require a complex WEDM cut and a large testing fixture to perform the test. The unidirectional single axis of loading required to both prepare and test a MSS sample provides increased potential for automation and this study further represents the maturity and use case for MSS in situations where UTT is difficult or impossible.

The small volumes probed by MSS also have the potential to produce property estimates that are substantially different from standard bulk tests (e.g., Sample number 4 in the present work). This is especially the case when the sample exhibits significant heterogeneity at the tested length scales. However, Khosravani et al. [18] presented the capability of indentation techniques to perform a multiresolution characterization of a hierarchical material system by collecting indentation measurements with a wide range of indenter tip radii for examining the length scales of heterogeneity, and by obtaining a sufficiently large set of statistically meaningful measurements. This work further represents that heterogeneity in MSS test results is unavoidable but that the rapid rate and low cost of testing allows for increased data collection and a better understanding of this heterogeneity and how it affects the bulk properties of SLM Inconel 718.

Currently, UTT-based protocols are often used to qualify AM builds using witness samples which are built alongside the part(s). However, the property measurements extracted from these samples are not likely to accurately reflect the properties of the AM- 
produced final part. Crocker [45] detailed the testing standards which are used to qualify metal AM parameters and materials and stated that there are unavoidable variations in thermal history between witness samples and the part being built. These variations in thermal history lead to property variations between the witness samples and the final AM product. MSS may be used to map the modulus and yield strength values across the cross-section of an AM-produced component and thereby produce a greater understanding of property variations throughout the part. The small testing volume and abundance of data collected from the MSS samples used in this study demonstrated that accurate property mapping is possible in SLM Inconel 718. Because of its simplicity, there is also the potential that MSS can be used to test an AM build in situ, which may then be used to provide valuable insights to improving the quality of a build during the AM process. MSFC-SPEC-3717 [46] is an example of current practices for qualifying an AM process, and it requires an array of tests including 15 tensile tests and metallographic evaluations. Since MSS can be performed directly on metallography samples as confirmed by this study, with the continued development and validation of MSS protocols, it is possible to dramatically reduce the number of required tensile tests.

\subsection{SPT}

SPT is a destructive test but has clear advantages over UTT. SPT requires a small volume of material, with samples obtained by simple straight cut machining, and exhibits a strong potential for high-throughput automation. As a result, there is tremendous potential for cost and effort savings compared to UTT, which requires larger material sample volumes. As shown in Table 2, a calculation of the machining and testing cost for SPT, using the experimental setup from this study, showed the cost of one SPT to be approximately $17 \%$ of the cost to machine and test one UTT sample.

Table 2. Cost comparison between SPT and UTT.

\begin{tabular}{ccc}
\hline Sample Processing Cost and Time & SPT & Tensile \\
\hline Machining Cost for 18 Samples (\$) & 112.5 & 562 \\
Machining Time for 18 Samples (hours) & 1.25 & 7.5 \\
Cost per Test (\$) & $<2$ & 115 \\
\hline
\end{tabular}

A calculation of the time savings for SPT vs UTT showed similar results. These cost and time savings can be greatly increased through the improvement of the SPT fixture design for automated high-throughput SPT.

Because of the use of the Swift equation for the characterization of the strain hardening response of the material, it is possible to estimate the values of yield strength and UTS from the best-fit values of the plasticity parameters, $K, \varepsilon_{0}$, and $n$. Yield strength may be predicted by using a plastic strain of 0.002 in Equation (14). Wang et al. [47] determined that UTS value can be estimated by applying the Considère criterion on the material constitutive response defined by the Swift equation.

The values of yield strength and UTS recovered from SPT measurements are compared against the corresponding UTT values in Figure 9.

The table summarizes the percent variation between the average SPT estimated yield strength and UTS values and the corresponding values measured in UTT.

This figure shows that the variation between the average SPT predicted yield strength and UTT yield strength for each sample was no greater than 5\%. It also shows that the variation between the average SPT estimated UTS and the corresponding measurement from UTT was no greater than $6 \%$ for all of the samples tested in this work.

Build parameters and post-build processing parameters often vary between AM builds and even within a single build. This study was aimed at evaluating these variations in both HIP and non-HIP samples. In the present work, HIPed samples 4 and 8 showed higher average UTS and yield strengths compared to the non-HIPed samples. This result was seen in both the standard UTT measurements as well as the SPT measurements. Therefore, the 
results obtained in this work demonstrated the ability of SPT to produce reliable estimates of both yield strength and UTS, which are typically the most important properties used in optimizing the performance of the final product.

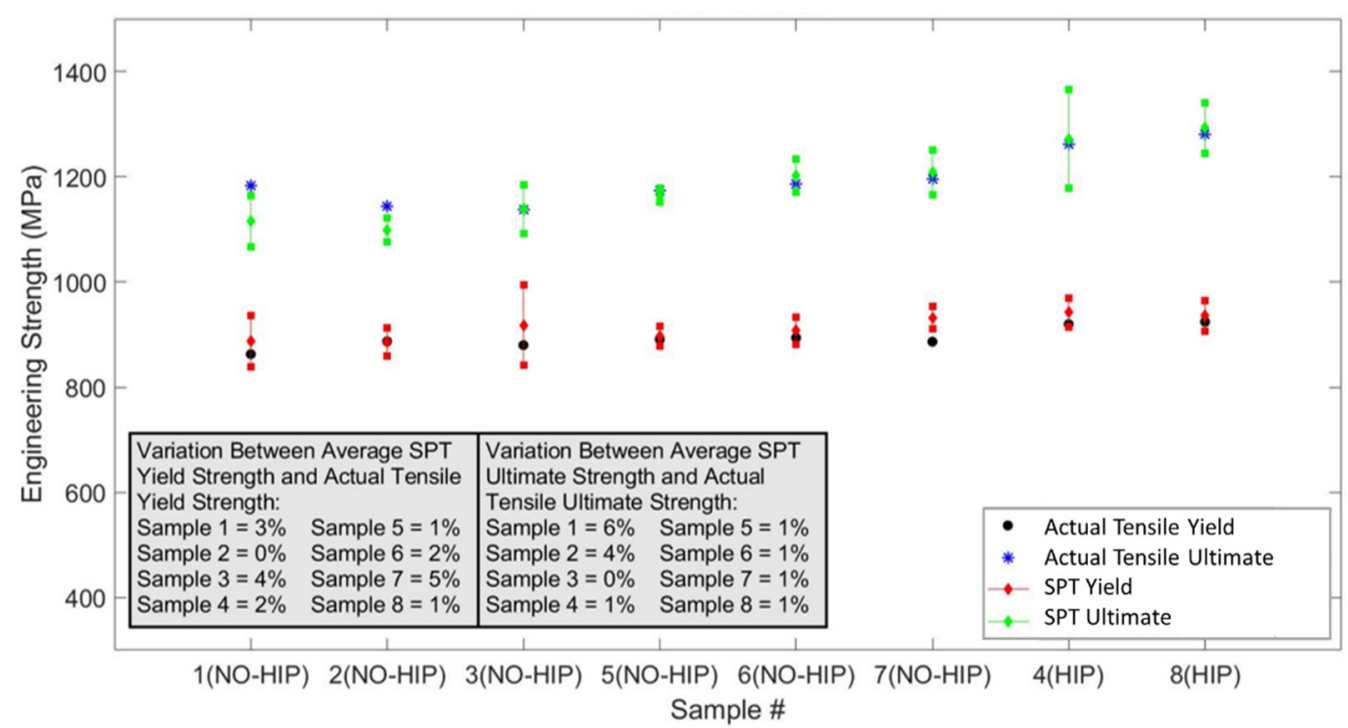

Figure 9. Yield strength and ultimate tensile strength (UTS) results from all SPTs showing average and standard deviations, along with the corresponding UTT measured yield strengths and UTS results.

\subsection{Relationship between UTT and Combined MSS and SPT}

The MSS protocols have been found to produce reliable measurements of the stressstrain response of the material in the initial elastic regime and up to small plastic strains (about $0.5 \%$ plastic strain). On the other hand, the SPT protocol was found to reliably estimate the stress-plastic strain response of the material (characterized using the Swift equation). These observations open the opportunity to combine these protocols to recover a stress-strain curve for the material that could be compared directly with the UTT measurement. As already discussed, the main advantages of MSS and SPT protocols are that they have tremendous potential for dramatic savings in cost and effort while requiring only small material volumes.

The combined stress-strain curve estimated from the MSS-SPT protocols is compared with the direct measurement from UTT Sample number 7 in Figure 10.

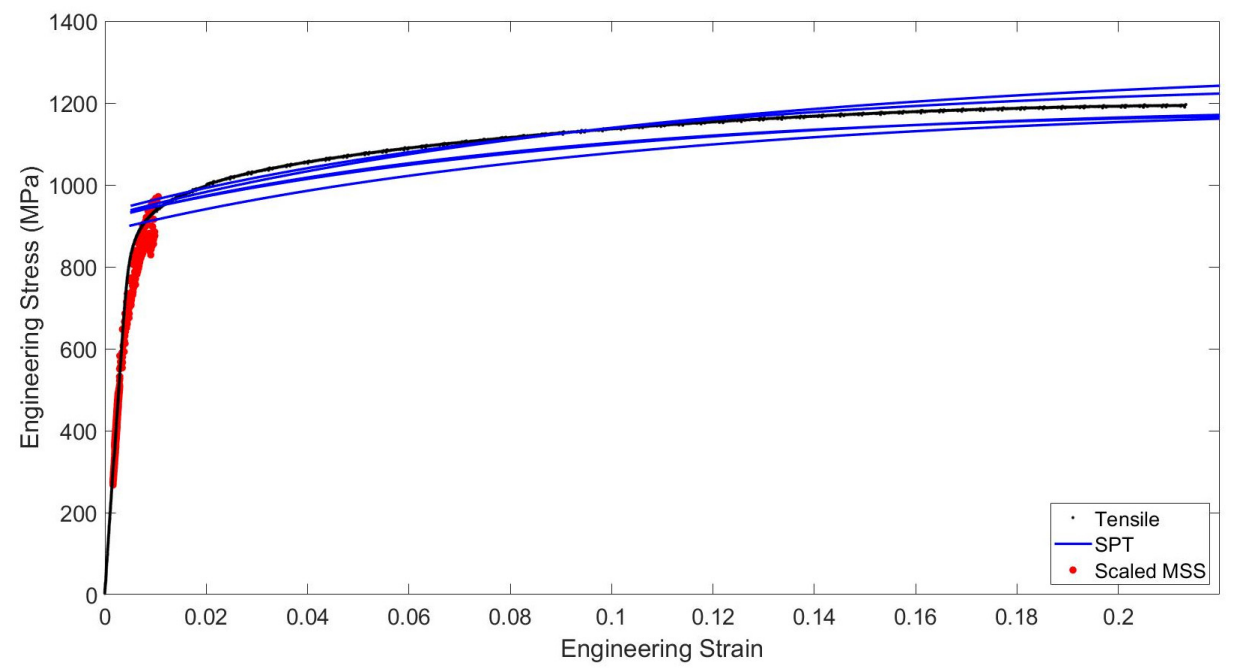

Figure 10. A graph showing both MSS and SPT stress-strain curves from Sample number 7 overlaid on the corresponding UTT stress-strain curve. 
The MSS and SPT stress-strain curves were combined graphically after the MSS curves were scaled (see Equations (10)-(13)) and both MSS and SPT stress-strain curves were converted from true stress-strain curves to engineering stress-strain curves for comparison with UTT measurements. The scaled MSS data is shown in greater detail in Figure 5.

The compact nature of the indentation machine used for MSS and SPT is well suited for materials and processes development on-board an orbiting laboratory such as the International Space Station (ISS). MSFC-SPEC-3717 and MSFC-SPEC-3716 [46,48] requires AM metallography samples to qualify AM parameters and processes, and these samples are of sufficient size to both provide metallographic imaging and the small amounts of material which are required for MSS and SPT. SPT samples are essentially slices of material no greater than $500 \mu \mathrm{m}$ so 3-5 SPT samples may easily be extracted from a metallography sample before it is mounted. The mounted and polished metallography samples may then be used for imaging and are highly conducive to MSS. The reduced material required for both SPT and MSS, as compared to UTT, allows for reduced costs to extract the combined MSS-SPT stress-strain response which can be used to determine modulus, yield strength, and ultimate strength. Lancaster et al. [49] performed fatigue testing using SPT on Ti-6Al$4 \mathrm{~V}$ and ascertained that the small sample volumes required for SPT can drastically reduce cost without compromising the accuracy of a fatigue test. Parvinian et al. [43] performed a trade study on the cost comparison between MSS and UTT and evidenced that both reduced material and testing costs associated with MSS drastically reduce its cost compared to UTT. This overall reduced cost for MSS and SPT is particularly advantageous when high-cost materials, such as high entropy alloys, bulk metallic glasses, or refractory metals, need alloy or processing development. The cost reduction and potential for automated high-throughput testing using MSS and SPT make it possible to produce a larger dataset of yield, modulus, and ultimate strength values for a lower cost and shorter time period than UTT.

\section{Conclusions}

As the need for lower cost products from rockets to cars rises, AM of metals must mature to meet this increase in demand. AM has the ability to drastically reduce the cost and time to manufacture a product while maintaining quality. While AM provides increased design flexibility and efficiency, it is still subject to the requirements necessary to qualify AM materials and processes. A paramount need for this qualification is a validation of mechanical properties which vary due to AM materials parameters and post-build processing. This is currently done using UTT and requires significant time and cost to determine the necessary mechanical properties such as yield strength, ultimate strength, elastic modulus, fatigue life, and ductility.

MSS and SPT are both processes which have been the subject of research for more than 30 years but are now beginning to develop protocols which can consistently determine intrinsic mechanical properties. The protocols for MSS and SPT described in this paper have demonstrated the capacity to track variations in intrinsic mechanical properties in prior research studies and further evidence of this has been demonstrated by producing stress-strain responses which could be used to observe property variations caused by postAM build heat treatment. The MSS-SPT stress-strain data used in this paper was collected using a small fraction of the time, cost, and material volume which was used to extract the same yield strength, ultimate strength, and modulus data found using UTT. Future work must build on FE and experimental SPT protocols to accurately predict ductility data so MSS and SPT can truly encompass the intrinsic material properties determined by UTT.

MSS and SPT are ideally suited for the significant parameter and process development requirements for AM and are highly capable of being automated for high-throughput data collection. As SPT and MSS are adopted by industry and a database of test results is established, they can be used to qualify materials and processes. This acceleration in AM materials and process development will increase the range of design and manufacturing competences to meet increasing demand. 
Author Contributions: Conceptualization, Z.S.C.; data curation, Z.S.C. and N.P.L.; formal analysis, Z.S.C., N.P.L. and H.N.K.; funding acquisition, S.R.K.; investigation, Z.S.C., N.P.L. and H.N.K.; methodology, Z.S.C. and H.N.K.; project administration, S.R.K.; resources, S.R.K.; software, N.P.L.; supervision, S.R.K.; Writing —original draft, Z.S.C., N.P.L., H.N.K. and S.R.K.; writing-review and editing, Z.S.C., N.P.L., H.N.K. and S.R.K. All authors have read and agreed to the published version of the manuscript.

Funding: This research was funded by the full-time degree seeking program at the NASA Marshall Space Flight Center and by the Office of Naval Research, grant number N00014-18-1-2879.

Institutional Review Board Statement: Not applicable.

Informed Consent Statement: Not applicable.

Data Availability Statement: The raw data required to reproduce these findings are available to download from [INSERT PERMANENT WEB LINK(s)]. The processed data required to reproduce these findings cannot be shared at this time as the data also forms part of an ongoing study.

Acknowledgments: The first author would like to appreciatively acknowledge support from the National Aeronautics and Space Administration (Marshall Space Flight Center) full-time degree seeking program. N.P.L., H.N.K., and S.R.K. acknowledge support from N00014-18-1-2879. The authors are thankful to Almambet Iskakov, Ali Khosravani, Soumya Mohan, and Natalia E. Millan of the Georgia Institute of Technology for their help with various aspects of the experiments reported in this work. The authors would also like to thank Ryan Wilkerson of the NASA Marshall Space Flight Center for review and editorial support.

Conflicts of Interest: The first author is employed as a full-time equivalent civil servant at the National Aeronautics and Space Administration. The views expressed in this paper do not necessarily represent the views of NASA.

\section{References}

1. Attaran, M. The rise of 3-D printing: The advantages of additive manufacturing over traditional manufacturing. Bus. Horiz. 2017, 60, 677-688. [CrossRef]

2. Jared, B.H.; Aguilo, M.A.; Beghini, L.L.; Boyce, B.L.; Clark, B.W.; Cook, A.; Kaehr, B.J.; Robbins, J. Additive manufacturing: Toward holistic design. Scr. Mater. 2017, 135, 141-147. [CrossRef]

3. Gibson, I.; Khorasani, A.M. Metallic Additive Manufacturing: Design, Process, and Post-Processing; Multidisciplinary Digital Publishing Institute, 2019.

4. Waller, J.M.; Parker, B.H.; Hodges, K.L.; Burke, E.R.; Walker, J.L. Nondestructive evaluation of additive manufacturing. Natl. Aeronaut. Space Adm. 2014.

5. Trosch, T.; Strößner, J.; Völkl, R.; Glatzel, U. Microstructure and mechanical properties of selective laser melted Inconel 718 compared to forging and casting. Mater. Lett. 2016, 164, 428-431. [CrossRef]

6. Babuska, T.F.; Wilson, M.A.; Johnson, K.L.; Whetten, S.R.; Curry, J.F.; Rodelas, J.M.; Atkinson, C.; Lu, P.; Chandross, M.; Krick, B.A.; et al. Achieving high strength and ductility in traditionally brittle soft magnetic intermetallics via additive manufacturing. Acta Mater. 2019, 180, 149-157. [CrossRef]

7. Kok, Y.; Tan, X.; Wang, P.; Nai, M.; Loh, N.; Liu, E.; Tor, S. Anisotropy and heterogeneity of microstructure and mechanical properties in metal additive manufacturing: A critical review. Mater. Des. 2018, 139, 565-586. [CrossRef]

8. Seifi, M.; Gorelik, M.; Waller, J.; Hrabe, N.; Shamsaei, N.; Daniewicz, S.; Lewandowski, J.J. Progress Towards Metal Additive Manufacturing Standardization to Support Qualification and Certification. JOM 2017, 69, 439-455. [CrossRef]

9. Gradl, P.; Brandsmeier, W.; Calvert, M.; Greene, S.; O’Neal, D.; Protz, C.; Richard, J.; Morgan, K. Additive manufacturing overview: Propulsion applications, design for and lessons learned. 2017.

10. Clinton, R., Jr. NASA's in Space Manufacturing Initiative and Additive Manufacturing Development and Quality Standards Approach for Rocket Engine Space Flight Hardware. Addit. Manuf. Def. Aerosp. 2016, 29-30.

11. Magagnosc, D.J.; Ligda, J.P.; Sano, T.; Schuster, B.E. Femtosecond Laser Machining of Micro-tensile Specimens for High Throughput Mechanical Testing. In Nonlinear Structures and Systems, Volume 1; Springer Nature: London, UK, 2017; Volume 5, pp. 7-9.

12. Aboulkhair, N.T.; Maskery, I.; Tuck, C.; Ashcroft, I.; Everitt, N.M. The microstructure and mechanical properties of selectively laser melted AlSi10Mg: The effect of a conventional T6-like heat treatment. Mater. Sci. Eng. A 2016, 667, 139-146. [CrossRef]

13. Rodriguez, R.A.; Gutiérrez, I. Correlation between nanoindentation and tensile propertiesInfluence of the indentation size effect. Mater. Sci. Eng. A 2003, 361, 377-384. [CrossRef]

14. Pathak, S.; Shaffer, J.; Kalidindi, S. Determination of an effective zero-point and extraction of indentation stress-strain curves without the continuous stiffness measurement signal. Scr. Mater. 2009, 60, 439-442. [CrossRef] 
15. Abendroth, M.; Kuna, M. Determination of deformation and failure properties of ductile materials by means of the small punch test and neural networks. Comput. Mater. Sci. 2003, 28, 633-644. [CrossRef]

16. Calaf Chica, J.; Bravo Díez, P.M.; Preciado Calzada, M. A new prediction method for the ultimate tensile strength of steel alloys with small punch test. Materials 2018, 11, 1491. [CrossRef] [PubMed]

17. Patel, D.K.; Kalidindi, S.R. Correlation of spherical nanoindentation stress-strain curves to simple compression stress-strain curves for elastic-plastic isotropic materials using finite element models. Acta Mater. 2016, 112, 295-302. [CrossRef]

18. Khosravani, A.; Morsdorf, L.; Tasan, C.C.; Kalidindi, S.R. Multiresolution mechanical characterization of hierarchical materials: Spherical nanoindentation on martensitic Fe-Ni-C steels. Acta Mater. 2018, 153, 257-269. [CrossRef]

19. Weaver, J.S.; Khosravani, A.; Castillo, A.; Kalidindi, S.R. High throughput exploration of process-property linkages in Al-6061 using instrumented spherical microindentation and microstructurally graded samples. Integr. Mater. Manuf. Innov. 2016, 5 , 192-211. [CrossRef]

20. Fernández, M.; Rodríguez, C.; Belzunce, F.J.; García, T.E. Use of small punch test to estimate the mechanical properties of sintered products and application to synchronizer hubs. Met. Powder Rep. 2017, 72, 355-360. [CrossRef]

21. Abendroth, M. FEM Analysis of Small Punch Tests. Key Eng. Mater. 2017, 734, 23-36. [CrossRef]

22. ASTM-E8, E. Standard test methods for tension testing of metallic materials. In Annual Book of ASTM Standards; American Society for Testing and Materials: West Conshohocken, PA, USA, 2001.

23. ASTM-E10-12. E10-12: Standard Test Methods for Brinell Hardness of Metallic Materials; American Society for Testing and Materials: West Conshohocken, PA, USA, 2012.

24. Kalidindi, S.R.; Pathak, S. Determination of the effective zero-point and the extraction of spherical nanoindentation stress-strain curves. Acta Mater. 2008, 56, 3523-3532. [CrossRef]

25. Donohue, B.R.; Ambrus, A.; Kalidindi, S.R. Critical evaluation of the indentation data analyses methods for the extraction of isotropic uniaxial mechanical properties using finite element models. Acta Mater. 2012, 60, 3943-3952. [CrossRef]

26. Pathak, S.; Kalidindi, S.R. Spherical nanoindentation stress-strain curves. Mater. Sci. Eng. R Rep. 2015, 91, 1-36. [CrossRef]

27. Abendroth, M.; Soltysiak, S. Assessment of Material Properties by Means of the Small Punch Test. In Recent Trends in Fracture and Damage Mechanics; Springer Nature: London, UK, 2015; pp. 127-157.

28. Manahan, M.; Argon, A.; Harling, O. The development of a miniaturized disk bend test for the determination of postirradiation mechanical properties. J. Nucl. Mater. 1981, 104, 1545-1550. [CrossRef]

29. Wolfenden, A.; Mao, X.; Shoji, T.; Takahashi, H. Characterization of Fracture Behavior in Small Punch Test by Combined Recrystallization-Etch Method and Rigid Plastic Analysis. J. Test. Eval. 1987, 15, 30. [CrossRef]

30. García, T.; Rodriguez, C.; Belzunce, F.J.; Suárez, C. Estimation of the mechanical properties of metallic materials by means of the small punch test. J. Alloys Compd. 2014, 582, 708-717. [CrossRef]

31. CWA-15627. CWA 15627: 2006 E, "Small Punch Test Method for Metallic Materials", PART A: A Code of Practice for Small Punch Creep Testing; CEN: Brussels, Belgium, 2006.

32. Bruchhausen, M.; Austin, T.; Holmström, S.; Altstadt, E.; Dymacek, P.; Jeffs, S.; Lancaster, R.; Lacalle, R.; Matocha, K.; Petzová, J. European standard on small punch testing of metallic materials. In ASME 2017 Pressure Vessels and Piping Conference; American Society of Mechanical Engineers: New York, NY, USA, 2017.

33. Leclerc, N.; Khosravani, A.; Hashemi, S.; Miracle, D.B.; Kalidindi, S.R. Correlation of Measured Load-Displacement Curves in Small Punch Tests with Tensile Stress-Strain Curves. Acta Mater. 2021, 204, 116501. [CrossRef]

34. Moussa, C.; Hernot, X.; Bartier, O.; Delattre, G.; Mauvoisin, G. Evaluation of the tensile properties of a material through spherical indentation: Definition of an average representative strain and a confidence domain. J. Mater. Sci. 2013, 49, 592-603. [CrossRef]

35. Chen, Y.H.; Park, S.U.; Wei, D.; Newstadt, G.E.; Jackson, M.A.; Simmons, J.P.; De Graef, M.; Hero, A.O. A Dictionary Approach to Electron Backscatter Diffraction Indexing. Microsc. Microanal. 2015, 21, 739-752. [CrossRef]

36. Yabansu, Y.C.; Iskakov, A.; Kapustina, A.; Rajagopalan, S.; Kalidindi, S.R. Application of Gaussian process regression models for capturing the evolution of microstructure statistics in aging of nickel-based superalloys. Acta Mater. 2019, 178, 45-58. [CrossRef]

37. Kleemola, H.J.; Nieminen, M.A. On the strain-hardening parameters of metals. Met. Mater. Trans. A 1974, 5, 1863-1866. [CrossRef]

38. Zarzour, J.F.; Konkol, P.J.; Dong, H. Stress-strain characteristics of the heat-affected zone in an HY-100 weldment as determined by microindentation testing. Mater. Charact. 1996, 37, 195-209. [CrossRef]

39. Kim, H.N.; Mandal, S.; Basu, B.; Kalidindi, S.R. Probing Local Mechanical Properties in Polymer-Ceramic Hybrid Acetabular Sockets Using Spherical Indentation Stress-Strain Protocols. Integr. Mater. Manuf. Innov. 2019, 8, 257-272. [CrossRef]

40. Clijsters, S.; Craeghs, T.; Moesen, M.; Kruth, J.P. Optimization of thin wall structures in SLM. In Proceedings of the Direct Digital Manufacturing Conference, Berlin, Germany, 14-15 March 2012.

41. Czotscher, T.; Wielki, N.; Vetter, K.; Vollertsen, F.; Meyer, D. Rapid Material Characterization of Deep-Alloyed Steels by Shock Wave-Based Indentation Technique and Deep Rolling. Nanomanuf. Metrol. 2019, 2, 56-64. [CrossRef]

42. Tsai, P.; Flores, K.M. High-throughput discovery and characterization of multicomponent bulk metallic glass alloys. Acta Mater. 2016, 120, 426-434. [CrossRef]

43. Parvinian, S.; Yabansu, Y.C.; Khosravani, A.; Garmestani, H.; Kalidindi, S.R. High-Throughput Exploration of the Process Space in 18\% Ni (350) Maraging Steels via Spherical Indentation Stress-Strain Protocols and Gaussian Process Models. Integr. Mater. Manuf. Innov. 2020, 9, 199-212. [CrossRef] 
44. Coll, G.T.; Webster, G.; Pankiewicz, O.; Schlee, K.; Aranyos, T.; Nufer, B.; Fothergill, J.; Tamasy, G.; Kandula, M.; Felt, A.; et al. Satellite Servicing Projects Division Restore-L Propellant Transfer Subsystem Progress 2020. In Proceedings of the AIAA Propulsion and Energy 2020 Forum, 24-28 August 2020.

45. Crocker, L. Literature Review on Tensile Test Standards for Metal Additive Manufacturing; National Physical Laboratory: Middlesex, UK, 2019.

46. NASA-MSFC. Specification for Control and Qualification of Laser Powder Bed Fusion Metallurgical Processes; NASA: Washington, DC, USA, 2018.

47. Wang, M.; Pang, J.; Qiu, Y.; Liu, H.; Li, S.; Zhang, Z. Tensile Strength Evolution and Damage Mechanisms of Al-Si Piston Alloy at Different Temperatures. Adv. Eng. Mater. 2018, 20, 1700610. [CrossRef]

48. NASA-MSFC. Standard for Additively Manufactured Spaceflight Hardware by Laser Powder Bed Fusion in Metals; NASA: Huntsville, AL, USA, 2018.

49. Lancaster, R.; Jeffs, S.; Illsley, H.; Argyrakis, C.; Hurst, R.; Baxter, G. Development of a novel methodology to study fatigue properties using the small punch test. Mater. Sci. Eng. A 2019, 748, 21-29. [CrossRef] 